\title{
XVIII.
}

Aus dem Landkrankenhause Cassel.

\section{Ein Beitrag zur Pathologie der Polyneuritis.}

\author{
Von \\ Dr. W. Rosenblath. \\ (Mit 5 Abbildungen im Text.)
}

Das Krankheitsbild der Polyneuritis, obwohl durch eine grosse Zahl casuistischer Mittheilungen und auch durch nicht wenige anatomische Untersuchungen unserem Verständnisse näher gerückt, enthält doch sicherlich in seiner klinischen wie pathologiseh-anatomischen Seite Züge, die noch wenig studirt sind. Einen kleinen Beitrag zur näheren Kenntniss dieses Krankheitsbildes glaube ich mit der Mittheilung der folgenden Fälle bieten zu können. Der erste (Walper) wird durch das Ungewöhnliche der Entwicklung und des Verlaufes in seinem Anfangsstadium Interesse erregen, der zweite (Pletzer - Alkohol-Neuritis) hat im Verlauf nichts Besonderes, konnte aber, da er tödtlichen Ausgang nahm, zum genauen Studium der histologischen Veränderungen benutzt werden. Der dritte gehört, streng genommen, nicht hierher. Doch schliesst er sich insofern eng an die vorigen an, als er klinisch als Neuritis diphtherica gelten musste, während die peripherischen Nerven anatomisch intact, die Rïckenmarkswurzeln dagegen schwer verändert gefunden wurden.

I. Adam Walper, 46 Jahre alt, Schneider aus Cassel.

Eltern starben an unbekannter Krankheit. Keine Nervenkrankheiten in der Familie. Patient machte 1870 den Feldzug mit, acquirirte damals Typhus, erholte sich aber wieder vollkommen und war gesund bis vor mehreren Jahren, wo er hier und da, besonders bei Aufregungen an „nervösen Zuckungen" gelitten haben will. 189014 Tage lang Influenza mit Fieber, Frost nnd Husten. März 1891 hatte er Gelbsucht, und die Beine schwollen an. Nach 4 Wochen Besserung, and nun war er gesund bis zum Mai 1892. Um diese Zeit erkrankte er mit Stichen in der Herzgegend und starker Anschwellung, die an den Füssen und Beinen begann und sich dann über den ganzen Körper ausdehnte. Der ihn behandelnde 
Arzt constatirte grosse Blässe, allgemeines Anasarka, ein Geräusch am Herzen und Stauungsharn. Mehrere Wochen später bemerkte Patient, dass Arme und Beine schwächer wurden, bald konnte er nicht mehr allein stehen und gehen und musste gefüttert werden. Zugleich stellte sich Zittern in Armen und Beinen und Parästhesien daselbst ein. Patient hatte oft das Gefübl von Ameisenkriechen, und als ob er mit Nadeln gestochen würde. Eigentliche Schmerzen dagegen hat er nie gehabt, ebensowenig Kopfweh, Erbrechen und Steifgkeit in der Wirbelsäule. Etwa im Juni verschwanden die Schwellungen allmählich, und nun bemerkte Patient sowohl wie seine Frau eine hochgradige Abmagerung der Hände, und dass die Finger sich krümmten. Mit dem Beginn der Krankheit bemerkte seine Umgebung weiterhin eine Aenderung in dem psychischen Verhalten des Patienten. Er wurde vergesslich und scheint hier und da verwirrt gewesen za sein, so dass er beim Versuche, seine Arbeit als Schneider fortzuführen, mehrfach verkehrte Arbeiten ausfübrte.

Im September 1892 suchte er das Landkrankenhaus auf. Die Untersuchung ergab:

Grosser Mann, mit reichlichem Fettpolster, mässige Blässe der Schleimbäute. Ziemliche Apathie, das Gedächtniss hat erheblich gelitten, doch lässt sich im Uebrigen kein höherer Grad von Demenz constatiren. Einfache Rechenaufgaben löst er richtig. Die höheren Sinnesfunctionen ungestört. Pupillen gleichweit, reagiren prompt. Bewegung der Bulbi ungestört, Augenhintergrund ohne Befund.

Musculatur des Gesichtes frei von Atrophien, beiderseits gleich innervirt, die Bewegungen von leichtem Zittern begleitet. Zunge kommt gerađe heraus, keine Schluckbeschwerden. Der weiche Gaumen symmetrisch. Rachenreflex vorhanden. Sprache ein wenig häsitirend.

Thorax breit. Wirbelsäule ohne Verbiegung und nirgends druckempfindlich. Bauchdecken fettreich, Musculatur des Stammes nicht atrophisch. Bewegungen des Rumpfes ungestört, Blase und Mastdarm intact. Reflexe des Stammes vorhanden und beiderseits gleich. - Lunge ohne Befund. Herzdämpfung klein, Puls regelmässig, von mittlerer Füllung und Spannung. Herztöne rein. Unterer Leberrand unter dem Rippenbogen tastbar. Milz nicht vergrössert. Urin frei von Eiweiss und Zucker.

Musculatur des Schultergiirtels ohne deutliche Atrophien, ebenso die des Oberarmes, die 'sich aber sehr schlaff anfühlt. Alle Bewegungen hier mit entsprechender Kraft ausführbar.

Vorderarme mager. Wenn der linke Vorderarm in die Höhe gehoben wird, sinkt die Hand schlaff herunter. Gegend der Mm. interossei und des Daumenballens deutlich abgemagert. Der Daumen steht in Opposition, die Grundphalangen gestreckt, die II. und III. Phalangen gebeugt, so dass die Hand, sich selbst überlassen, halb geschlossen ist. Streckung im Handgelenk ist aufgehoben, Bengung geschieht mit geringer Kraft, mit noch geringerer Adduction und Abduction. Extension des Daumens aufgehoben, Abduction und Adduction mit sehr geringer Kraft ausführbar.

Streckung der Finger gar nicht, Beugung und Spreizung nur sehr unvollkommen möglich.

An der rechten Hand sind die Veränderungen im Ganzen dieselben, nur nicht so hochgradig. Die Atrophie am Thenar und Hypothenar oder 
Interossei ist ähnlich wie links. Streckung der Hand ist hier in beschränktem Maasse ausführbar. Stellung und Function der Finger wie links.

Fibrilläre Zuckungen sind in der atrophischen Musculatur nur hier und da wahrnehmbar.

In der rechten Hand kann Patient zwischen Zeigefinger und Daumen mit Mühe einen Löffel halten.

Die Musculatur der Oberschenkel ist nicht atrophisch, fühlt sich auch ziemlich straff an, fibrilläre Zuckungen bestehen nicht. Am Unterschenkel ist die Wadenmusculatur sehr schlaff, aber nicht reducirt. Dagegen giebt sich die Atrophie der Peroneusmusculatur beiderseits durch eine deutliche Furche nach aussen von der oberen Tibiakante zu erkennen. Die Füsse, sich selbst überlassen, stehen in Spitzfussstellung. Die grobe Kraft ist erheblich reducirt. Am besten gelingt noch Beugung der Oberschenkel. Beugung und Streckung im Knie geschieht mit geringer Kraft und ruft Tremor in der Musculatur der Oberschenkel hervor. Bewegungen der Füsse und der Zehen sind fast gar nicht möglich, Gehen und Stehen nur mit kräftiger Unterstlitzung.

Die tactile Sensibilität, der Schmerz- und Temperatursinn sind iberall ungestört. Keine sichere Ataxie. - Patellarreflex fehlt beiderseits, ebenso der Plantarreflex.

Die elektrische Prüfung ergab folgendes Verhalten:

Faradisch war unerregbar der N. radialis beiderseits, schwer erregbar der N. median. und ulnaris über dem Handgelenk. Als unerregbar erwiesen sich bei directer Reizung des M. supinator long., Extens. carpi rad. et uln., digitor. comm. auf beiden. Seiten, während die Musculatur der Beugeseite prompt reagirte. Keine Zuckung gaben Abductor und Flexor pollic. brev., während Opponens reagirte, und auch von Abductor digiti min. schwache Zuckungen erhalten wurden.

An den unteren Extremitäten sind faradisch unerregbar der N. cruralis, tibialis und peroneus. Von Muskeln antwortet nur der Tensor fasciae, der Quadriceps und der Sartorius bei starken Strömen mit Zuckung. - Die galvanische Untersuchung liefert ganz ähnliche Resultate, und speciell fand sich im M. tibial. anticus und dem Extens. digitor. ped. comm. partielle Entartungsreaction.

Die Diagnose anlangend, so konnte nicht zweifelhaft sein, dass multiple Neuritis vorlag. Die Lähmung war eine symmetrische und mit Atrophie verbunden; Sensibilitätsstörungen fehlten, das Kniephänomen war geschwunden, in der Unterschenkelmusculatur fand sich partielle Entartungsreaction. Blase und Mastdarm war ungestört.

Die Auffassung, dass es sich hier um ein Leiden der peripheren Nerven handele, wurde durch den weiteren Verlauf der Krankheit bestätigt. Die Atrophien und mit ihnen die Lähmungen gingen nämlich allmählich zurück. Nach einigen Monaten konnte Patient die Arme and Hände besser gebrauchen, und er konnte allein geben und stehen. An den Beinen besserte sich zunächst die Beweglichkeit und 
Kraft der Oberschenkel, Beugung und Streckung im Knie, erst später ging die Peroneuslähmung zurück, so dass Patient lange Zeit den typischen „Steppergang" darbot. Das Kniephänomen war bei seiner Entlassung (Juli 1893) noch nicht auszulösen. Ebensowenig stellte sich die fribere geistige Regsamkeit wieder her. Patient blieb apathisch, hielt sich immer für sich und nahm an der Umgebung nur wenig Antheil.

Der Verlauf der Krankheit, soweit er das Nervensystem betrifft, bietet somit nichts Auffälliges. Was den Fall dagegen zu einem bemerkenswerthen macht, das ist die Aetiologie des Leidens und die Erscheinungen, unter denen es sich entwickelte. Von den Schädlichkeiten, welche bei uns zu Lande die Neuritis zu erzengen vermögen, war hier keine nachzuweisen. Weder war eine der bekannten Infectionskrankheiten vorausgegangen, noch spielten hier die Gifte speciell der Alkohol nicht -, an die man zu denken hat, eine Rolle, noch lag eine zur Neuritis disponirende mit Kachexie verlaufende Krankheit zu Grunde.

Dagegen setzte die Krankheit ein mit deutlicher Anämie, mit sehr hochgradigen Oedemen und Stauungsharn. Zugleich wurde ein Geräusch am Herzen gehört. Dieser Beginn im Verein mit den später sich zeigenden Lähmungen könnte nun den Gedanken aufkommen lassen, dass Polymyositis vorgelegen hätte. Demgegenüber genügt es indessen, darauf hinzuweisen, dass Schmerzen in der Musculatur niemals im Verlauf der ganzen Krankheit bestanden haben, und dass speciell die Oedeme in keiner Weise an die Musculatur sich anschlossen, sondern dass sie sich von den Füssen an aufwärts ausbreiteten. Wenn mir auch über den Befund am Herzen in jener Zeit nichts Näheres bekannt geworden ist, so genïgt doch die Angabe, dass ein Geräusch am Herzen gehört wurde, und Stauungsharn bestand im Verein mit der erwähnten Art der Ausbreitung vollkommen, um den Hydrops als einen durch Stauung bedingten zu kennzeichnen.

Für ein Leiden aber, das mit Anämie, Störung der Herzthätigkeit und Oedemen beginnt und dann zu atropbiseher Lähmung führt, liegt, soweit ich sehe, gar keine andere Analogie vor, als die Beriberi. Bekanntlich werden bei dieser Krankheit (vgl. Scheube, Die Beriberi-Krankheit im Arch. für klin. Medicin. XXXI u. XXXII) vier Formen unterschieden: die rudimentäre, die atrophische, die hydropische oder hydropisch-atrophisehe und die acute perniciöse. Die bydropisch-atrophische wäre es, die hier zum Vergleiche herangezogen werden darf. Unter Zeichen von Anämie, Herzklopfen, Oppressionsgefühl und verminderter Diurese entwickelt sich Schwäche meist zu- 
nächst der unteren Extremitäten, Anasarka und Hydropsien. Die rasch sich entwickelnde Atrophie der Musculatur wird durch die Oedeme verdeckt und oft ganz wie in dem beschriebenen Fall erst nach Zurückgehen der Oedeme entdeckt. Die elektrische Erregbarkeit der erkrankten Muskeln kann alle Grade der Störung aufweisen, und die Sehnenreflexe können fehlen. Ebenfalls wie in meinem Fall bleiben die Gesichtsnerven meist intact, während Störungen der geistigen Thätigkeit auch bei der Beriberi vorkommen. Weniger passend ist das Verhalten der Sensibilität. Während die Störung in meinem Falle sich lediglich auf Parästhesien beschränkte, sind bei der Beriberi meist alle Qualitäten der Empfindung beeinträchtigt. Sieht man von diesem Punkte ab, so besteht zwischen den hydropischatrophisehen Fällen der Beriberi und dem mitgetheilten eine völlige Congruenz, die sich auch auf den schliesslichen Ausgang in Genesung erstreckt, entsprechend der im Allgemeinen gitinstigen Prognose bei der Kakke.

Analoge Fälle scheinen in der deutschen Literatur der Polyneuritis bislang nicht verzeichnet $\mathrm{zu}$ sein. Nur eine Beobachtung von Adler $\left.{ }^{1}\right)$ muss hier erwähnt werden, weil der Autor dem mitgetheilten Krankheitsfalle eine Mittelstellung zwischen der Kakke und der Polymyositis einräumt. Hier entwickelte sich bei einem 36 jährigen Potator im Anschluss an Erbrechen und Durehfall, Dyspnoe und Cyanose unregelmässiger, kleiner Puls, Verbreiterung der Herzdämpfung, Ascites und Hydrothorax mit Leber- und Milzsehwellung. Gleichzeitig wurden unter Spannungsgefühl in den Muskeln und zunehmender Schwäche die Extremitäten ödematös, so zwar, dass dieselben Spindelform annahmen, und die Hände und Füsse frei blieben. Am rechten Oberarm liess sich deutlich constatiren, dass die Schwellung durch Volumzunahme der Muskeln bedingt war. Die geschwollene Musculatur ist auf Druck schmerzhaft, die Nerven nicht. Sensibilitätsstörungen objectiv nicht nachweisbar. Sehnenreflexe feblen. Elektrisch theils complete, theils partielle Entartungsreaction. Tod nach $2^{1 / 2}$ monatlicher Daner dureh Pericarditis.

Histologisch fanden sich zellige Infiltrate im intermusculären Bindegewebe, den kleineren Arterien anliegend. Die Nerven wiesen nur an den Muskelästchen Zerfall der Markscheiden anf.

In diesem Krankheitsbilde stimmen, wie der Autor hervorhebt, die Hydropsien, die Herzschwäche, die Lähmung und das elektrische Verbalten zur Kakke, während die Schwellung der Musculatur mehr zur Polymyositis gehört.

1) Deutsche med. Wochenschrift. 1894. Nr. 10. 
Dem gegenüber treten nun in meinem Falle die zur Polymyositis gehörenden Symptome vollständig zurück. Er ist insofern reiner, wenn man als Paradigma die Beriberi im Auge behält.

Senatorl) hat uns mit Fällen bekannt gemacht, welche lehren, dass zwischen der typischen Neuritis und Myositis Uebergangsformen existiren. Adler's Fall wie der meinige heben einen weiteren verwandtschaftlichen Zug hervor, indem sie zeigen, dass beide Krankheiten mit schweren Circulationsstörungen unter Betheiligung des Herzens sich verbinden können.

II. Frau Pletzer, 31 Jahre alt.

Seit längerer Zeit dem Alkohol ergeben, den sie in Form von Bier und Liqueur zu sich nimmt. Seit ungefähr 4 Wochen Schwäche in den Beinen. Sie knickt beim Gehen oft in den Knien ein, dass Treppensteigen wird ihr sauer. Zugleich stellen sich Schmerzen in den Beinen ein und das Gefïhl, als ob sie mit Nadeln gestochen würde. Seit 8 Tagen wurden auch die Arme schwach, und die Schwäche in den Beinen nahm so zu, dass Patientin ans Bett gefesselt wurde. Am Tage der Aufnahme entwickelte sich ein Delirium. - Vor $1 / 4$ Jahr hat sie abortirt.

Objectiv ergab sich: Mässiges Fettpolster, Gesicht etwas gedunsen und geröthet. Vollkommener Verwirrungszustand. Patientin vermag ihre Anfmerksamkeit nur auf ganz kurze Zeit zu concentriren, weiss nicht, wie lange sie sich in der Anstalt befindet, kann über ihre Krankheit keine zusammenhängende Auskunft geben. Sinnesfunctionen und Gebiet der iibrigen Hirnnerven ohne Störung.

Wirbelsäule ohne Befund. Musculatur der Bauchdecken schlaff. Patientin kann sich obne Unterstïtzung nicht aufsetzen und fällt im Bette zurück, sobald ihr die Stiitze entzogen wird. Reflexe am Stamm erloschen. Stuhl angehalten. Orin lässt sie unter sich.

Mit den oberen Extremitäten kann Patientin fast alle Bewegungen ausführen, aber mit geringer Kraft. Besonders geschwächt ist die vom N. radialis versorgte Musculatur beiderseits. Bei erhobenen Vorderarmen sinken die Hände in typischer Stellung herab. Atrophien bestehen nicht. Mechanische Erregbarkeit der Musculatur erhalten. Triceps-Reflex fehlt beiderseits.

An den Unter-Extremitäten ist die Musculatur der Oberschenkel, wie trotz des Fettpolsters deutlich, besonders an der Vorderfläche ausserordentlich reducirt. Deutlicher Muskelschwund der Peronealmusculatur. Weniger reducirt ist die Wadenmusenlatur. Sie fuihlt sich aber sehr schlaff an. Beide Beine befinden sich im Zustande fast vollkommen schlaffer Lähmung. Nur ganz geringe Bewegungen können mit den Zehen besonders des rechten Fusses ausgeführt werden.

Obliquus-, Plantar- und Patellar-Reflexe fehlen.

Feinere Prüfungen des Muskelsinnes der tactilen Sensibilität, des Thermalsinnes waren bei dem benommenen Zustande der Kranken undureh-

1) Ueber acute und subacute Neuritis und Myositis. Zeitschrift für klin. Medicin. XV. 
führbar, doch schien mir die tactile Sensibilität an den unteren Extremitäten herabgesetzt. Sicher war die Schmerzempfindung an vielen Stellen der unteren Extremitäten vermindert. Während Patientin anf Nadelstiche an Arm und Leib bald reagirt, lassen sich an den Beinen Hautfalten durchstechen, ohne dass eine Schmerzäusserung erfolgt.

Bei der elektrischen Prüfung ergab sich für Gesicht, Hals, Brust, Arme normales Verhalten. Nur im Radialis-Gebiet die Erregbarkeit für beide Stromesurten herabgesetzt. Erloschen war die Erregbarkeit für beide Stromesarten in der Musculatur des Bauches, der Oberschenkel, der Waden und des Peroneus rechts. Im Peroneusgebiet der linken Seite Anodenzuckung mit charakteristischem, trägem Charakter.

Schon am Tage nach der Aufnahme entwickelte sich Fieber, das bald über $39^{\circ} \mathrm{C}$. stieg. Der Puls warde schlecht, die Respiration frequent. An Stelle der Verwirtheit trat Benommenheit. Ueber dem Unterlappen der linken Lunge entwickelte sich eine Dämpfung, Dyspnoe und Cyanose trat hinzu, und sechs Tage nach der Aufnahme starb die Patientin.

Die Krankheit hatte also im Ganzen etwa 5 Wochen gedauert und war unter dem Bilde einer aufsteigenden Lähmung verlaufen. Doch war klar, dass nicht etwa eine sogenannte Landry'sche Lähmung vorlag, zu deren Bilde ja besonders die Atrophien, Entartungsreaction und Delirien nicht gehören. Es konnte vielmehr keinem Zweifel unterliegen, dass es sich hier um eine Polyneuritis handelte. Die symmetrische Ausbreitung, der atrophische und schlaffe Charakter der Lähmung, der Verlust der Reflexe, die gleichzeitige Alteration der Sensibilität u. s. w., alles dies wies auf einen peripheren Sitz der Lähmung hin.

Aetiologisch konnte mit Rücksicht auf den vorausgegangenen Abort nur flüchtig an die Möglichkeit einer puerperalen Neuritis gedacht werden. Denn einmal lag dieses Ereigniss beim Ausbruch der Krankheit schon 6-8 Wochen zuritck, und zweitens machten der zugestandene Missbrauch geistiger Getränke und besonders auch die Delirien die Auffassung der Neuritis als einer alkoholischen unabweisbar.

Unklar blieb in dem Krankheitsverlauf dann nur, ob die gegen das Ende hin sich entwickelnde Dyspnoe und Cyanose durch die Pneumonie oder eine Lähmung der Respirationsmusculatur bedingt war. Der später mitgetheilte Befund, der auch am Nervus phrenicus erhoben wurde, lässt wohl der Annahme Raum, dass hier auch degenerative Veränderungen in den Respirationsnerven mit im Spiel waren.

Bei der Section fand sich am Gehirn Oedem der Pia und Arachnitis chronica fibrosa. Die Substanz des Gehirnes und Rückenmarkes, sowie die peripheren Nerven obne erkennbare Veränderung. In der Spitze der rechten Lunge eine kirschgrosse Caverne mit käsigen Wan- 
dungen. Im Oberlappen der linken Lunge einige gruppenweise beisammenstehende käsige Herde; im Unterlappen frische Lobulärpneumonie. Leichte Fettleber, chronischer Magenkatarrh.

Zur mikroskopischen Untersuchung wurden in Müller'scher Flüssigkeit conservirt: das Rückenmark, beide Nn. crurales, ischiadici, peronei und radiales, je ein medianus, ulnaris und phrenicus; ferner Stiickchen der Musculatur der Extremitäten.

Die mikroskopisehe Untersuchung ${ }^{1}$ ) ergab nun Folgendes: Der Quersehnitt eines guten Carminpräparates lässt im Ganzen nur sehr wenige

\section{Fig. 1.}

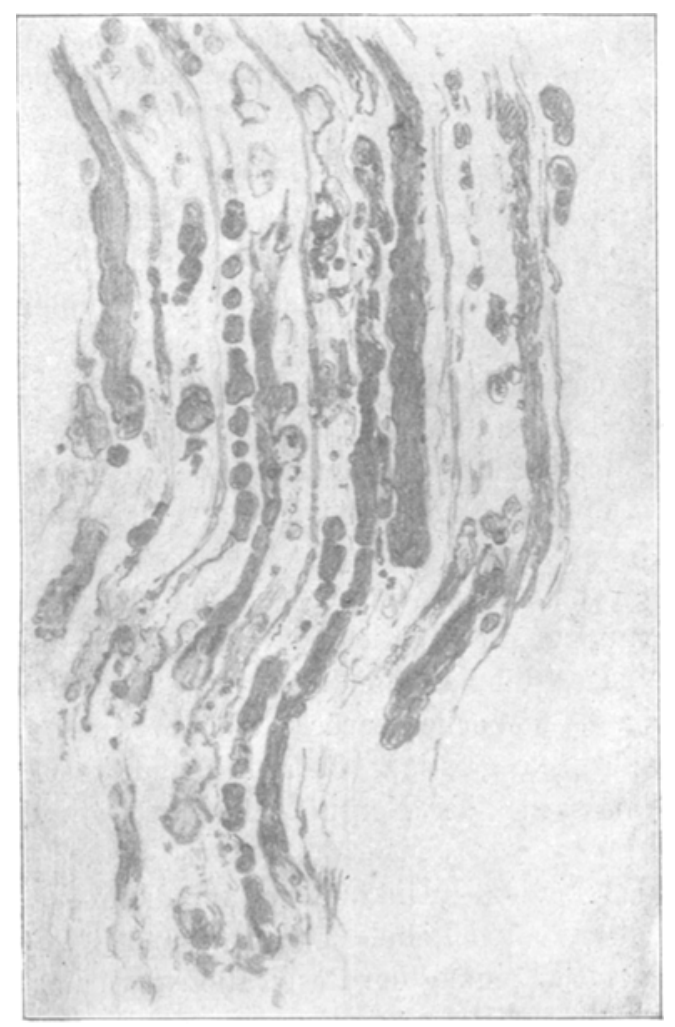

N. cruralis. Längssehnitt. Pal'sche Fürbung.

wohl erhaltene Axencylinder als runde, tief roth gefärbte Scheiben erkennen. Bei weitem die Mehrzahl ist hochgradig verändert. Verhältniss-

1) Für die Deutung mancher Befunde bin ich Herrn Prof. Marchand in Marburg, der eine Anzahl Präparate mit mir durchzusehen die Güte hatte, zu grossem Danke verpflichtet. 
mässig selten ist der Axencylinder im Ganzen stark gequollen und füllt als blassrothe Scheibe die Schwann'sche Scheide mehr oder weniger vollständig aus, hin und wieder von dieser noch durch eine ganz schmale ringförmige Zone getrennt. Nicht immer ist diese Scheibe homogen, sondern oft ganz unregelmässig gefärbt, birgt blasige Liicken oder ist von

Fig. 2-4.

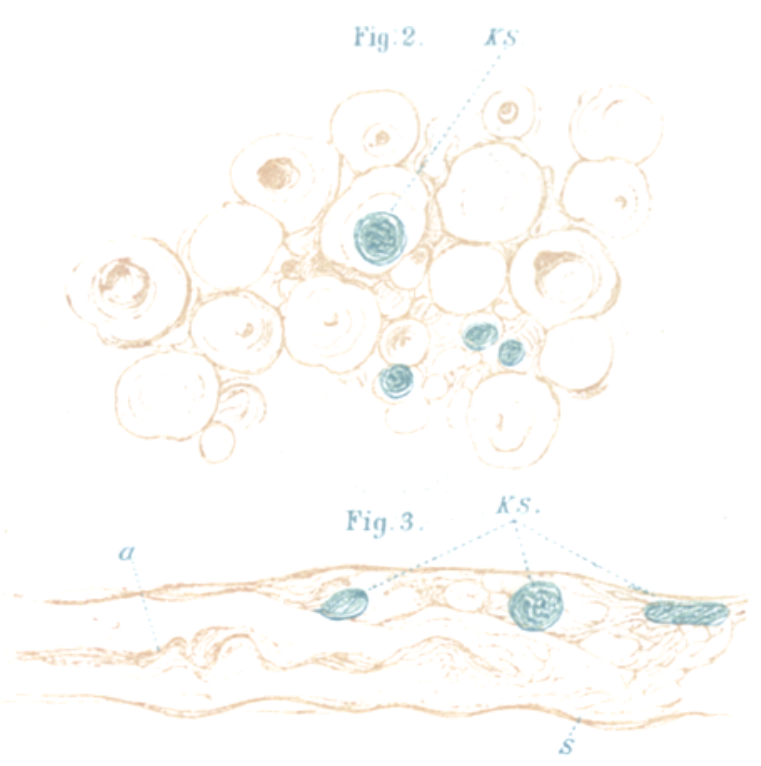

\section{Fig.4.}

Fig. 2. N. cruralis. Quersehnitt. Hämatoxylin-Eosin. Axencylinder in verschiedenen Stadien des Zerfalls. - Fi g. 3. N. cruralis. Längsschnitt. Hämatoxylin-Eosin. Links ein aufgelockerter und gewundener Axencylinder, rechts Zellen der Sohwann'schen Scheide. - Fig. 4. Faser aus dem N. cruralis, fast ganz ausgefiullt mit gewucherten Zellen der Sch wann'schen Scheide. Das Protoplasma dieser Zellen von anseheinend vacuolärer Beschaffenheit. Hämatoxylin-Eosin. $a=$ Axencylinder. $S=\mathrm{Sch}$ wa n n'sche Scheide. $K S=$ Kerne der S o h w a n'schen Scheide.

(Fig. 1 nach Zeiss DD., Fig. 2-4 nach Immersion Zeiss $1 / 12$ und Ocular III gezeichnet.)

körniger Beschaffenheit, wobei die körnigen Theile intensiv gefärbt sind. Oefter ist an Stelle des Axencylinders eine concentrisch geschichtete 
Scheibe von blasser Färbung zu sehen, oder an Stelle des Axencylinderquerschnittes tritt ein Netzwerk mit rundlicher Begrenzung und tiefroth gefärbten Fäden, einem Chromatingerüst eines Zellkernes nicht unähnlich. Die Grösse solcher Figuren ist oft beträchtlich, der Durchmesser mag den eines rothen Blutkörperchens um das Drei- bis Vierfache übersteigen. Hin und wieder findet man den Axencylinder anch aus seiner Richtung verlagert, gewunden oder quer in der Schnittebene verlanfend; an solchen Stïcken ist dann auch in der Regel eine Auffaserung zu bemerken. An der Mehrzahl der Fasern ist eine Scheidung in Mark und Axencylinder überhaupt nieht mehr zu vollziehen. Die Schwann'sche Scheide wird von einer homogenen oder leicht gekörnten Masse ausgefüllt, die sich mit Carmin nicht gefärbt hat. An Querschnitten, die mit Hämatoxylin und Eosin gefärbt sind (Fig. 2), siebt man nicht selten innerhalb der Schwann'schen Scheiden Kerne liegen, und zwar sind diese Kerne beträchtlich grösser als die vormalen, ragen weiter in das Lumen hinein, ja sie liegen manchmal vollkommen central an Stelle des Axencylinders, manchmal fiillen sie die sonst leere Schwann'sche Scheide vollkommen aus, und einzelne sind von einem grossen Protoplasmahofe umgeben. An solchen Fasern ist vom Axencylinder meist gar nichts mehr und von Markscheide meist nur an die Wand gedrängte Trümmer zu sehen.

Natürlich wird das Verhalten der Markscheide erst am WeigertPräparate völlig klar. Der Querschnitt desselben zeigt schon mit schwacher Vergrösserung, dass zwischen den einzelnen schwarz gefärbten Fasern grosse Zwischenräume mit gelbbrauner Färbung liegen. Stärker vergrössert finden sich auch die Fasern, deren Markscheiden schwarze Färbung angenommen haben, schwer verändert. Wirklich normale Fasern, bei denen die Markscheide als schwarzer Ring sich repräsentirt, sind nur wenige zu sehen. Oft ist der Ring in eine intensiv gefärbte körnige und eine blasse drusige Partie geschieden, oft füllt das Mark als Scheibe die ganze Schwann'sche Scheide aus oder liegt in Tropfen oder Tröpfchen zerklüftet in derselben. An allen Präparaten finden sich aber auch Fasern kleinsten Calibers, an denen von solchem Zerfall nichts zu sehen ist. Noch deutlicher enthüllen Längsschnitte, Fig. 3 und 4, die Schwere der Veränderungen. Am Hämatoxylin-Eosin-Präparat tritt schon bei schwacher Vergrösserung die starke Vermehrung der Kerne hervor. Dass diese sämmtlich dem Gewebe selbst angehören, lässt sich bei starker Vergrösserung leicht feststellen. Leukocyten fehlen vollständig. Die Kerne liegen nur zum Theil ähnlich wie in der Norm der Se h wann'schen Scheide an, nur dass sie durch kürzere Abstände von einander getrennt sind. Sie sind oval oder spindelig, von Protoplasma ist in ihrer Umgebung nicht viel zu sehen. Anders verbält sich ein anderer Theil. Dieses ist in den Binnenraum, der die Schwann'sche Scheide angrenzt, hineingertickt. Diese Kerne sind meist etwas blasser gefärbt, oval oder unregelmässig rundlich und sind von einem grossen Protoplasmahofe umgeben. Nicht selten sieht man einen Schlauch der Schwan n'schen Scheide, der auf eine Strecke ganz von solchen Zellen eingenommen ist. Vgl. Fig. 4. An solchen Stellen ist das Caliber der Nervenfasern meist weit; an anderen ist es eng, die leeren Scheiden scheinen sich fast ganz aneinander gelegt $z \mathfrak{u}$ haben. An Präparaten mit Kern- und starker Eosinfärbung gelingt es bei 
einiger Aufmerksamkeit, an vielen Stellen ganz wohl Protoplasma der gewucherten Zellen und Markscheide oder Reste derselben auseinander zu halten. Das Protoplasma hat sich mit Eosin deutlich gefärbt, das Myelin nicht, das Protoplasma ist homogen oder feingranulirt, das Myelin behält auch im Cauadabalsam meist etwas Glanz und zeigt im Inneren oft F̈altelungen oder netzförmige Figuren. Achtet man hierauf, so erkennt man an vielen Stellen die zerklifftete Markscheide deutlich und sieht, wie sich an vielen Stellen das Protoplasma von allen Seiten zwischen die Marktrümmer einschiebt und dieselben umfliesst. Hier und da finden sich Zellen von enormer Grösse, die aufgeblähte Markscheide auf weite Strecken ausfüllend. In dem Protoplasma solcher Zellen finden sich dann häufig Vacuolen oder tropfenähnliche Bildungen, Fig. 4, die durch Eosin nicht gefärbt sind. Ich glaube, dass die Deutung derselben als eingeschlossene Marktrümmer zulässig ist.

Auch die Axencylinder sind an derartigen Präparaten unschwer zu erkennen, Fig. 3. Nur selten sieht man einen, der scheinbar wohl erhalten ist. Bei den meisten erkennt man bald Auftreibungen und Anschwellungen, Zerkluffungen und Fragmentirung. Nicht selten findet man einen Axenfaden scheinbar intact. Verfolgt man denselben dann eine Strecke, so kommt man oft an eine Stelle, wo er mit einer kolbigen Auftreibung endigt. Ferner sieht man oft, manchmal, wie es scheint, im Inneren von Myelinklumpen aufgesplitterte oder korkzieherartig aufgewundene Fäden, die sich mit Eosin gut gefärbt haben und zweifelsohne Reste von Axencylindern darstellen.

Die Schwann'schen Scheiden selbst waren, soweit ich erkennen konnte, iiberall erhalten. An einzelnen Stellen lagen die Kerne besonders dicht, die Scheiden waren hier fast leer und eng, und die Kerne lagen spindelförmig neben und hintereinander.

Am Weigert-Präparat, Fig. 2, findet sich am Längsschnitt eine ganz ausserordentlich starke Zerklüftung des Markes, das sich in unregelmässigen Figuren, Flaschenform, Tropfen der verschiedensten Grösse repräsentirt. Gerade da, wo es in feinen Tropfen zerspalten ist, treten auch recht zahlreich Kerne zwischen den Markresten auf. Doch gelang mir gerade an Weigert- oder Pal-Präparaten die Kernfürbung nicht mehr so gut, wie ich erwartet hatte, jedenfalls, weil die Stücke inzwischen zu lange conservirt worden waren. Gerade diese Präparate geben daher iber das Verhalten der Zellen, des Protoplasma u. s. w. zu den Markresten keinen weiteren Aufschluss.

Diese beschriebenen Veränderungen scheinen den Nerven fast in seinem ganzen Verlaufe betroffen zu haben, wenigstens fand ich sie gleichmässig in den Stämmen und in den intramusculären Aesten.

Die Musculatur wurde an verschiedenen Stellen untersucht. Sie erwies sich jedoch als vollkommen unverändert. Weder im Zwischengewebe, noch an den Fasern selbst vermochte ich etwas Auffälliges zu entdecken.

Auch das Rückenmark bot normale Verhältnisse.

Die in der Literatur niedergelegten Befunde über histologische Veränderungen in den Nerven bei der Alkoholneuritis waren mir 
nicht alle zugänglich. ${ }^{1)}$ Soweit ich sehen kann, ist es hauptsächlich der Zerfall der Markscheide, der wiederholt an Weigert- oder Osmiumpräparaten studirt und beschrieben wurde. Veränderungen des Axencylinders wird öfters hervorgehoben, Betheiligung des Zwischengewebes meist vermisst. Verschiedene Autoren sprechen von Kernvermehrung, ohne dass man sicher erkennen kann, welcher Ablkunft dieselben sind. Einige Male wird das Vorkommen von Körnchenzellen erwähnt, und Gombault²) spricht von voluminösen Zellen, welche die Schwann'schen Scheiden zum Theil ganz ausfüllen und Marktrümmer in sich aufnehmen können. Babes beschreibt in dem Atlas der pathologischen Histologie des Nervensystems folgende Veränderungen der Nervenwurzeln bei Alkoholneuritis: Nervenfasern, die an einem Ende noch normale Verhältnisse bieten, schwellen im weiteren Verlaufe an, der Axencylinder wird blass, und in seiner Umgebung "differenzirt" sich das Mark, so dass der Axencylinder in ein grobkörniges Reticulum eingescheidet liegt. Der Axencylinder soll dann wellige und knäuelförmige Verlängerung und auch Fragmentirung erfahren; die Schlingen desselben sollen immer von einer "speciellen dünnen, körnigen Myelinscheide" umgeben sein. Babes fällt es auf, dass ein Theil der Myelinscheiden durch Pikrocarmin sich röthlich färbt, und es erklärt sich das als Folge theilweiser Aufblähung der chromatischen Substanz des Axencylinders.

Im Allgemeinen ist somit jedenfalls so viel sicher, dass die Alkoholneuritis weniger eine Entzündung als vielmehr eine Degeneration der Nerven darstellt. Die feineren Vorgänge bei diesem Process sind jedoch begreiflicher Weise, da immer nur einzelne Befunde vorliegen, die jedesmal nur bestimmte Phasen des Verlaufes erläutern, noch wenig bekannt. Man muss daher, um diese Befunde zu verstehen, besser bekannte, einigermaassen analoge Vorgänge heranziehen, und besonders dürften hier die Veränderungen der Nerven infolge von Durchschneidungen in Betracht kommen. Diese Veränderungen sind in neuerer Zeit besonders eingehend von v. Büngner ${ }^{3}$ ), später von $S$ tr öbe u. A. untersucht und stellen sich nach dem ersteren Autor, in Kürze wiedergegeben, folgendermaassen dar: Die augenfälligste Veränderung zeigt sich am frühesten an der Markscheide. Diese verliert ihre gerade Begrenzungslinie, ist stellenweise aufgetrieben, stellenweise verschmächtigt. Daran schliesst sich Segmentirung und Zerklüftung, zunächst zu gröberen Bruchstücken, später zu kleineren mehr tropfenähnlichen

1) Die neue Arbeit von Köpp en im Archiv für Psychiatrie kam mir leider erst nach Abschluss dieser Arbeit zu Gesicht. Anm. b. d. Correctur.

2) Comptes rend. T.112. 3) Ziegler, Beiträge zur path. Anatomie. Bd. X. 
Gebilden. Ebenso wird bald der Axencylinder theils verschmächtigt, theils anfgetrieben und aufgelockert, seine Continuität aufgehoben, die Fragmente zerfasert oder korkzieherartig aufgewunden. Ist seine Zerkliftung in discontinuirliche Elemente vollendet, so können die Marktropfen zwischen den Bruchstiicken zusammenfliessen und Theile des Axencylinders einschliessen. Bald macht sich Volumszunahme der Kerne in den Schwann'schen Seheiden bemerkbar, und es treten Mitosen in ihnen auf. Zugleich nimmt das Protoplasma dieser Zellen beträchtlich zu, und weiterhin schieben sich diese wuchernden Zellen in das Innere der Schwann'sehen Seheiden hinein, ihr proliferirendes Protoplasma breitet sich zwischen den Marksegmenten aus, umfliesst die Trümmer der Markscheide und des Axencylinders und nimmt diese in sich auf. Diese Zellen wirken also als Phagocyten. Bei diesem ganzen Vorgange spielen Leukocyten gar keine Rolle. Die weitere Sehilderung, besonders der Neubildung der Nervenfasern, kann ich für den vorliegenden Fall ausser Acht lassen.

Vergleiche ich diese Resultate mit dem, was die Untersuchung. des vorliegenden Falles lebrt, so findet sich eine weitgehende Uebereinstimmung in den wesentlichen Punkten. Die Zerklüftung des Markes und der Axencylinder sind auch hier angenfällige und wohl auch die frühzeitigsten Veränderungen. Dann findet sich die Wucherung der Kerne der Sehwann'schen Scheide, die sich in starker Vermehrung und auch in Vergrösserung der Kerne und Proliferation ibres Protoplasmas kundgiebt. Auch hier rücken diese Zellen von der Wand der Schwann'schen Scheide in das Innere derselben, umfliessen die Marktrümmer und nehmen auch Segmente des zerfallenden Axencylinders in sich auf. Auch hier vollziehen sich diese Veränderungen ohne Betheiligung des Blut-Bindegewebsapparates und ohne Einwanderung von Leukocyten. Dentliche Zeichen von Regeneration der Fasern dagegen konnte ich nicht nachweisen.

\section{III.}

Die $\sqrt{ }$ rage nach dem anatomischen Substrat der im Gefolge der Diphtherie auftretenden Lähmungen ist jedenfalls der Untersuchung noch sehr bedürftig. Auch wenn wir uns auf die Betrachtung derjenigen Lähmungen beschränken, die meist als dureh Veränderung der peripheren Nerven bedingt aufgefasst werden, die Hemiplegie nach Diphtherie also von vornherein ausgeschlossen wird, so bieten die bisher erhobenen pathologiseh-anatomischen Befunde doch noch eine ziemliche Mannigfaltigkeit. Da finden sich verzeichnet: Blutungen von verschiedener Grösse im Gehirn, Oblongata, Rückenmark und Nerven, 
Veränderungen in der grauen Substanz des Rückenmarkes, entzündliche Veränderungen im interstitiellen Gewebe der Nerven, Degeneration der Nerven selbst, Entzündung und Degeneration der Muskeln; auch völlig negative Befunde sind mitgetheilt. Daraus ergiebt sich die Aufforderung, vorkommenden Falles die Gelegenheit zu histologischer Untersuchung derartiger Fälle nicht voribbergehen zu lassen.

Marie Gundlach, 8 Jahre alt, aufgenommen den 22. September 1895. Am 16. September erkrankt mit Halsschmerzen; am 19. September von dem behandelnden Arzte 600 I.-E. Heilserum injicirt.

Bei der Aufnahme diffuser Belag auf beiden Tonsillen, Uvula und Gaumenbögen. Kieferdrïsen links geschwollen. Temp. 38,40. Puls frequent, regelmässig 120. Athmung frei. Im Urin Spuren von Eiweiss. Der Ernährungszustand des Kindes war ein guter. Das Allgemeinbefinden war nicht schlecht.

In der Zeit vom 24.-30. September stösst sich der Belag ab, Fieber besteht nicht mehr, der Puls geht herunter, bleibt aber klein. Urin enthält mehr Eiweiss.

4. October. Oedem der unteren Extremitäten. Puls sehr klein, Herzdämpfung wenig nach rechts verbreitert, systolisches Geräusch an der Spitze. Urin enthält etwa $3 / 4$ Proc. Eiweiss, Nierenepithelien, hyaline und Epitheleylinder.

In den folgenden Tagen verbreitert sich die Herzdämpfung mehr, der Spitzenstoss rückt über die Maramillarlinie hinaus. Hier und da wird statt des systolischen Geräusches ein doppelter Ton gehört. In dieser Zeit ist der Patellarreflex noch auslösbar, wenn auch manchmal nicht sofort.

Am 20. October sind die Patellarreflexe beiderseits erloschen, und von jetzt an treten die nervösen Störungen in den Hintergrund.

25. October. Patientin verschluckt sich, Sprache nasal, Rachenreflex erloschen.

27. October. Parese und ausgeprägte Ataxie der Beine. Das Kind vermag sich auch mit kräftiger Unterstïtzung nicht auf die Beine zu stellen, und zwar hauptsächlich weil es die Beine in unzweckmässige Position bringt. Grobe Kraft der oberen Extremitäten gleichfalls vermindert, scheinbar auch geringe Ataxie. Sensibilität, soweit zu prüfen, scbeint unbetheiligt.

30. October. Seit heute Morgen die Respiration mühsam und frequent. Die linke Seite bleibt deutlich in der Athmung zurück. Leichte Cyanose. Puls klein und frequent. Athemgeräusch vesiculär nur links hinten abgeschwächt. - Aus dem Munde fliesst fast continuirlich Speichel $\mathrm{ab}$, der augenscheinlich nicht verschluckt werden kann. Beim Aufrichten des Kindes sinkt der Kopf schlaff auf die Seite oder hintenüber. Vollständige Aphonie. Abends Temperatursteigerung auf 38,40. Linke Seite steht völlig still. Speichel fliesst nicht mehr ab, Patientin schluckt etwas und kann sich mit leiser Stimme vernehmlich machen.

31. October. Athmung mühsamer, Speichel fliesst ab, völlige Aphonie, Cyanose. Grosse Schwäche. Exitus. 
Albuminurie hatte in den letzten Wochen abgenommen, bestand aber bis zum Ende. Das Sediment war gering. Im Befunde des Herzens hatte sich nichts geändert.

Auf die Frage, ob der üble Ausgang durch eine höhere Antitoxindosis hätte vermieden werden können, gehe ich hier nicht ein, möchte aber bemerken, dass das Kind in die Anstaltsbehandlung erst am 6. Krankheitstage eintrat, also zu einer Zeit, in der die Serumbehandlung nicht mehr sehr wirksam ist. Zudem bestand in den beiden folgenden Tagen leidliches Woblbefinden, und die localen Erscheinungen besserten sich; zugleich fiel das Fieber ab, und unter diesen Umständen wurde von einer weiteren Injection abgesehen. $\mathrm{Ob}$ es weiterhin angezeigt ist, Folgezustände der Diphtherie, speciell die Lähmungen mit Serum zu behandeln, darüber liegen meines Wissens noch keine Erfahrungen vor.

Klinisch konnte die Beurtheilung des Falles nicht zweifelhaft sein. Die Lähmung hatte mit Schluckbeschwerden und Verlust des Kniephänomens eingesetzt; dann hatte sich hochgradige Ataxie der unteren Extremitäten und bald grosse Schwäche der gesammten Musculatur bei scheinbar intacter Sensibilität angeschlossen. Während gleichzeitig Niere und Herz schwer geschädigt waren, führte Lähmung der Athmungsmusculatur besonders linksseitig bei zeitweilig fast völlig aufgehobenem Schluckvermögen das Ende herbei.

Lähmung der Schlund- und Respirationsmusculatur mit Ataxie der unteren Extremitäten und Westphal'schem Zeichen musste zur Diagnose "Neuritis" führen.

Die Ataxie scheint mir nach den Erfahrungen, die ich in den letzten Jahren bei Beobachtung von etwa 600 Diphtheriefällen machen konnte, nächst der Gaumenlähmung die häufigste Nervenstörung zu sein, und man bekommt hier alle Grade der Ataxie, von der leichtesten bis zur schwersten zu sehen. In vielen Fällen tritt sie in die Erscheinung als eine in wenigen Tagen vorübergehende Unsicherheit im Gehen und Stehen, bei der man im Zweifel sein kann, ob eine wirkliche Ataxie vorliegt, oder ob die Kinder nur infolge der Bettlage die Herrschaft über die Beine verloren haben. Gleichzeitiger oder nachfolgender Verlust des Kniephänomens wird in solehen Fällen die Auffassung der Störung beeinflussen. In schweren Fällen kam immer eine deutliche Parese hinzu. Das Verhalten der Sensibilität war wechselnd, doch wurde in den leichten Fällen nur über Schmerzen in den Beinen geklagt. Fast immer waren die Schlnckbeschwerden die ersten hervortretenden Lähmungssymptome. In dem mitgetheilten Falle erreichten sie gegen das Ende eine derartige Höhe, dass das Kind über- 
haupt nichts mehr zu schlucken vermochte, und der reichlich abgesonderte Speichel ähnlich wie im letzten Stadium der Bulbärparalyse zum Munde herausfloss. Auch auf dies Symptom ist schon einige Male hingewiesen. Der Uebergang der Lähmung auf die Respirationsmusculatur schliesslich ist bei verschiedenen Formen der Neuritis bekannt und gefürchtet.

Wenn somit auch zweifellos der mitgetheilte Fall unter diejenigen gehört, die als Neuritis bei Diphtherie geführt werden, so ist es doch noch zweifelhaft, ob derartige Fälle wirklich anatomisch durch Neuritis bedingt sind, und von einer Kenntniss der specifischen Veränderungen, die speciellen Störungen zu Grunde liegen, sind wir noch weit entfernt. Reine Fälle von Ataxie sind meines Wissens noch nicht anatomisch untersucht, und ebenso ist noch unaufgeklärt, worauf der nun seit längerer Zeit bekannte Schwund des Kniephänomens bei der Diphtherie beruht. Es mag hier beiläufig erwähnt werden, dass ich in 3 Fällen, in denen die Kinder, die im Anschluss an Diphtherie das Kniephänomen eingebüsst hatten und an Lobulärpneumonien starben, die Cruralnerven mit der W eiger't'schen Methode, mit Hülfe von Kernfärbungen und an Osmiumpräparaten untersucht habe mit durchaus negativem Erfolge. Ich hatte also um so mehr Grund, die Untersuchung des vorliegenden Falles nicht auf das periphere Nervensystem zu beschränken.

Bei der Section fand sich das Nervensystem makroskopisch ohne Veränderung.

Dilatation beider Herzventrikel und grosser Thrombus im linken Ventrikel. In der Milz ältere Infarctnarben. Gallenblase enthält einen grossen Stein. Nieren makroskopisch kaum verändert, nicht vergrössert, Rinde gelblich. Mesenterialdrïsen verkäst. Enteritis follicularis.

Die nähere Untersuchung des Nervensystems bezog sich anf Gehirnrinde, Oblongata, Rückenmark und Phrenicus, Vagus, Cruralis, Ischiadicus. Zur Fixation wurden benutzt: Alkohol, Sublimat, Fle m m in g'sche Lösung, M ii l l r'sche Flüssigkeit. Die so vorbereiteten Organe wurden nach den Methoden von Nissl, Pal, Marchi, sowie mit den gewöhnlichen Kernfärbungen weiter bearbeitet.

Die Untersuchung der Ganglienzellen der Centralwindungen und der grauen Substanz des Rüickenmarkes ergab ein negatives Resultat. Besonders die motorischen Zellen der Vorderhörner liessen weder in der Structur der Kerne, noch der des Zellenleibes oder der Fortsätze irgend eine Abnormität erkennen. Negativ blieb fernerhin die Untersuchung der peripheren Nerven, besonders auch des Vagus und Phrenicus, abgesehen von vereinzelten Blutungen in der Scheide des letzteren. Deutliche Veränderungen liessen sich dagegen in den Rückenmarkswurzeln feststellen. Auf diese Befunde wurde ich zuerst aufmerksam an Präparaten, die nach Marchi behandelt waren. Es hoben sich hier nämlich an Rückenmarks- 
querschnitten, die nach Marchi behandelt waren, und die die Wurzeln mit umfassten, schon bei Lupenvergrösserung schwarze Flecke, gerade in der Einstrahlungszone der hinteren Wurzel gelegen, hervor. Im Halsmark waren solche Stellen am reichlichsten anzutreffen, am ausgeprägtesten in den hinteren Wurzeln, Fig. 5, doch fehlten dieselben auch in den vorderen nicht. Im unteren Hals- und Brustmark waren sie nur spärlich, reichlicher wieder im Lendenmark. In den hinteren Wurzeln des Halsand Lendenmarkes, die für sich eingebettet, in Längsschnitte zerlegt und nach Pal untersucht wurden, liess sich in einzelnen Btindeln Zerfall der Markscheiden nachweisen, während die grosse Mehrzahl normale Verhältnisse darbot.

Fig. 5.

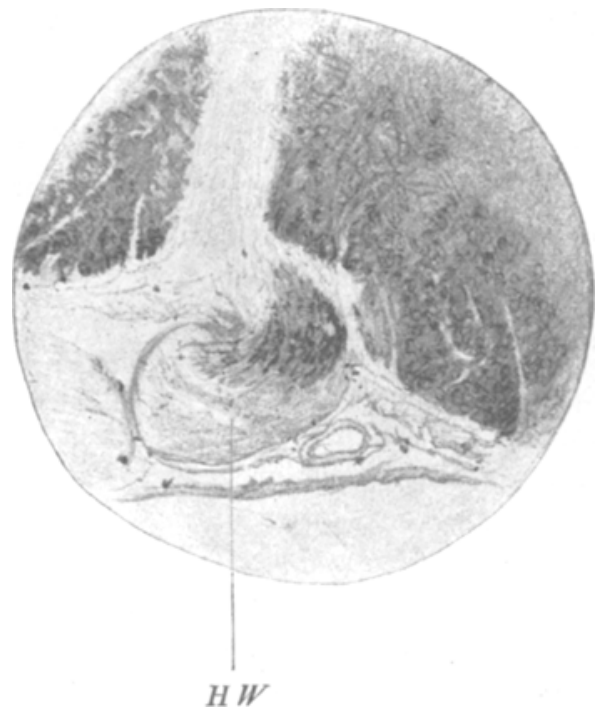

Halsmark. Marchi-Präparaț. Eintrittsstelle der hinteren Wurzel. Sehwache Vergrösserung.

Auch im Rïckenmark sind deutliche Veränderungen sichtbar, allerdings nur mit der Methode Marchi's nachweisbar. Zunächst fällt an derartigen Präparaten eine Schwärzung der Randzone auf, die fast den ganzen Querschnitt umgreift, zwischen den Hinterhörnern am ausgeprägtesten ist, ebenso in der Gegend der Kleinhirnseitenstrangbahn stark hervortritt und längs des Sulcus longit. anter. fast verschwindet. In der Lissauer'schen Randzone ist gar keine Schwärzung eingetreten. Weiterhin heben sich im Halsmark die ans den hinteren Wurzeln einstrahlenden Fasern durch ihre dunklere Färbung deutlich von den übrigen ab. Besonders sind es Fasern groben Calibers entweder in den Burdach'schen Strang einstrahlend oder zu den Bogenbündeln gehörig in das Hinterhorn eintretend, die, oft intensiv schwarz gefärbt, bis tief in das Hinterhorn oder den Hinterstrang hinein verfolgt werden können. Ebensolche dunkelschwarz gefärbte Fasern finden sich in der vorderen Commissur und auch 
besonders zahlreich in den die Vorderstränge durchbrechenden Wurzelfasern. Weniger deutlich, aber doch sicher vorhanden, ist die Veränderung in den aus der Gegend der motoriseben Zellen kommenden und die graue Substanz nach der Wurzelaustrittszone hin durchsetzenden Fasern. Uebrigens fanden sich auch ziemlich gleichmässig durch die weisse Substanz verstreut an den meisten Schnitten mehr schwarze Fasern als dem normalen Verhalten entsprechen dürfte. An vielen der erwähnten Fasern lässt sich bei stärkerer Vergrösserung auch deutlicher Zerfall des Markes constatiren.

Von Muskeln wurde nur das Zwerchfell untersucht. Es bot normale Verhältnisse.

In den Nieren fand sich der bei Diphtherie gewöhnliche Befund des körnigen Zerfalles der Drüsenepithelien, daneben aber auch herdweise Infiltration und Verbreiterung des Zwischengewebes $z$ wischen den gewundenen Harnkanälchen und in der Cmgebung der Glomeruli.

Der erhobene Befund weicht also insofern von manchen anderen mitgetheilten $a b$, als er die Unversehrtheit des peripheren Nervensystems nachwies. Die pathologischen Veränderungen sitzen hier besonders in den Rückenmarkswurzeln und denjenigen Gebieten des Riuckenmarkes, welche $\mathrm{zu}$ diesen Wurzeln in näheren Beziehungen stehen. Nun ist ja von vornherein wohl kaum zu erwarten, dass bei allen diphtherischen Lähmungen gleiche Veränderungen nachgewiesen werden, und insofern hat ja die schon erwähnte Mannigfaltigkeit der Befunde nichts Ueberraschendes. Sehen wir doch auch z. B. beim Alkohol, dass er einmal das periphere Nervensystem, dann wieder das Rückenmark oder das Gehirn allein oder vorzugsweise schädigt. Die Aufgabe ist vielmehr, die Bedingungen festzustellen, unter denen dieser oder jener Theil des Nervensystems vorzugsweise entartet, und nachzuforschen, ob aus der Zahl der bei Diphtherie vorkommenden Lähmungen sich klinisch besondere Gruppen herausheben lassen, deren Eigenthümlichkeiten während des Lebens schon eine bestimmte Localisation der pathologischen Veränderungen vermuthen lassen.

Für den vorliegenden Fall liegt es nun nahe, die während des Lebens beobachtete Ataxie aus der beschriebenen Erkrankung der hinteren $W$ urzeln abzuleiten. Aehnliche Beobachtungen existiren bereits zwei.

Bikeles ${ }^{1)}$ untersuchte in einem Falle von Ataxie und hochgradiger Schwäche nach Diphtherie das Nervensystem. Er fand die peripheren Nerven intact, am Rückenmark dagegen mit der Methode von Marchi: Degeneration in den Wurzeln, den hinteren mehr als den

1) Zur pathologischen Anatomie der postdiphth. Lähmung. Arbeiten aus dem Institut für Anat. u. Physiol. des Centralnervensystems an der Wiener Universität 1894. Heft II. 
vorderen, und die Veränderungen liessen sich entsprechend dem intramedullären Verlauf der Wurzelfasern längs dem Hinterhorn bis in die grane Substanz hinein verfolgen. Die Lissauer'sche Randzone ist auch hier unverändert.

Anders liegen die Verhältnisse in einem von $\operatorname{Preisz}^{1}$ ) mitgetheilten Falle, bei dem während des Lebens atactischer Gang und grosse Schwäche notirt ist. Preisz fand vordere und hintere Wurzeln überall degenerirt, am stärksten jedoch im Lendenmark linkerseits, im Rückenmark den linken Goll'schen Strang von der Lende bis zum Halsmark, den rechten nur im Halsmark entartet. Dazu kommt im Lendenmark ein degenerirtes Gebiet, welches der Abbildung nach ungefähr der medialen hinteren Wurzelzone Flechsig's entsprechen würde. Diese Veränderungen konnten am WeigertPräparat erboben werden.

Beide Autoren sind der Ansicht, dass die constatirten Veränderungen die Ataxie bedingt haben. Die Verschiedenheit beider Befunde liegt auf der Hand. Gemeinsam ist ihnen die Entartung der hinteren Wurzeln. Aber in dem von Bikeles beschriebenen Falle, wo die Entartung der Wurzeln sich auf die Hinterstränge fortsetzt, entsprechend dem Verlauf der medial gelegenen gröberen hinteren Wurzelbündel, besteht eine greif bare Analogie mit der Tabes. In dem Falle von Preis z dagegen bestehen Verhältnisse, die jede Vergleichung mit dieser Krankheit verbieten, denn es fehlt die Degeneration in der eigentlichen Wurzelzone ganz, und das im Lendentheile halbseitig degenerirte Feld entspricht dem, welches bei der Tabes frühzeitig nicht befallen wird. Da diese Krankheit aber bislang die einzige mit Ataxie verlaufende ist, deren Anatomie gründlich studirt ist, so wird man einstweilen Bedenken tragen mïssen, so ganz anders gestaltete Befunde, wie sie in dem Falle Preisz vorliegen, zur Erklärung der Ataxie zu verwenden.

Mit dem Falle von Bikeles hat der meinige Vieles gemeinsam: das Fehlen der Veränderungen in den peripheren Nerven, die Hauptbetheiligung der Wurzeln, und hier wieder vorwiegend der hinteren, die Fortsetzung der Degeneration der hinteren Wurzeln in die Wurzelzone und in die graue Substanz. Behält man die Analogie mit der Tabes im Auge, so ist das Freibleiben der Lissauer'schen Randzone in beiden Fällen nicht ausser Acht zu lassen. Auch muss es für meinen Fall Bedenken erregen, dass die Veränderungen in den

1) Beiträge zur Anatomie der diphther. Lähmungen. Deutsche Zeitschrift für Nervenheilkunde. Bd. VI. 
hinteren Wurzeln des Halsmarkes am hochgradigsten gefunden wurden, während die Ataxie am ausgeprägtesten die unteren Extremitäten befallen hatte. Immerhin wäre es denkbar, dass bei weiterer Untersuchung des Lendenmarkes nach Marchi (es wurde nur eine Scheibe dieser Region nachgesehen) noch schwerere Entartung aufgefunden wäre. Ueberspringt doch auch der pathologische Process bei der Tabes hier und da eine Wurzelhöhe. Indessen sind weitere Erfahrungen hier abzuwarten. Von besonderem Interesse wiurde es natïrlich auch sein, wenn in Fällen, die ohne Ataxie verlaufen, die hier erwähnten Gebiete der hinteren Wurzeln intact gefunden würden.

Die Entartung der vorderen Wurzeln wird man wohl ohne Widerspruch mit der allgemeinen Parese der Glieder in Verbindung bringen dürfen, und hier ist besonders von Interesse, dass an manchen Fasern dieser Process bis in die Nähe der Vorderhornzellen verfolgt werden konnte, während die Zellen selbst, nach Niss I behandelt, keine Veränderung erkennen liessen.

Welcher Natur die in der vorderen Commissur liegenden degenerirten Fasern sind, vermag ich nicht zu entscheiden. Ebenso möchte ich über die Bedeutung der Schwärzung der Randzone kein bestimmtes Urtheil abgeben. 